\title{
Experimental Studies on Output, Spatial, and Spectral Characteristics of a Microdroplet Dye Laser Containing Intralipid as a Highly Scattering Medium
}

\author{
Hiroshi Taniguchi, Shinji Tanosaki, Kazuhiro Tsujita, and Humio Inaba, Fellow, IEEE
}

\begin{abstract}
Lasing characteristics of Rhodamine 6G dyedoped microdroplets containing highly scattering fat emulsion Intralipid-10\% are studied experimentally. Noteworthy findings are that well-defined lasing threshold can be observed and one order or more magnitude enhancement of emission intensity with suitable (optimum) conditions of the Intralipid mixing ratio, in comparison with original neat-dye lasing microdroplets. We present and discuss the measured results of input-output intensities for different dye concentrations and dye-Intralipid mixing ratios in this high-gain laser dye-soft scatterer system and microscope images of spatial distribution of light emission from both the microdroplets containing neat-dye and dyeIntralipid mixture. It was found that almost no-lasing neat-dye microdroplets, which have either much higher or much lower dye concentration, can achieve lasing by substituting suitably certain amounts of the Intralipid, causing multiple light scattering. Spectral measurements of lasing outputs from the Rhodamine 6G dye-Intralipid microdroplets show the tendency of the disappearance of the well-known mode structures, owing to the morphology-dependent resonances of this microspherical cavity inherent to the neat-dye microdroplets. It is our belief that the present results make this novel method of dye-Intralipid microsystem very attractive for a variety of future applications, including diagnostic tools for highly sensitive detection and identification of small quantity objects and species embedded or hidden in highly scattering media, such as biological tissues and cellular structures.
\end{abstract}

\section{INTRODUCTION}

$\mathbf{S}$ TUDIES on the interaction of laser beams with transparent micrometer-sized spherical droplets (microspheres or microdroplets) have shown that they behave as optical cavities with a high quality factor $(Q$ value) at discrete characteristic frequencies corresponding to specific size-towavelength ratios. The ratios are defined as the size parameter $x=2 \pi a / \lambda$, where $\lambda$ is the optical wavelength and $a$ is the radius of the microsphere [1].

A number of papers on lasing from microspheres such as dye-doped liquid microdroplets [2]-[4] and active crystals [5], dye-doped solid spheres [6], [7], and on stimulated Raman

Manuscript received March 21, 1996; revised June 26, 1996. This work was supported in part by the Ministry of Education, Science, Sports, and Culture of Japan through Grant (C) 07650041.

H. Taniguchi and S. Tanosaki are with the Department of Electrical and Electronic Engineering, Faculty of Engineering, Iwate University, Morioka 020, Japan.

K. Tsujita is with Biophotonics Information Laboratories, Yamagata 990, Japan.

H. Inaba is with the Tohoku Institute of Technology, Taihaku-Ku, Sendai 982, Japan.

Publisher Item Identifier S 0018-9197(96)08038-4. scattering (SRS) [8]-[10] from liquid microdroplets irradiated by intense laser beams were first reported over ten years ago. These lasing and SRS emissions occur at discrete wavelengths corresponding to morphology-dependent resonances (MDR's) (or to the whispering-gallery mode) of the microsphere. Previous investigations have experimentally suggested that more efficient laser performance owing to MDR's is possible in liquid microspheres than in solid ones because of lower optical losses in the former [11], [12]. From the viewpoint that microdroplets must contain particulates, lasing behaviors were studied recently in liquid droplets containing a fluorescent dye solution of mixed fluorescent sol [13], polystyrene spheres [14], and undoped latex-sol particles [15].

On the other hand, current investigations have reported the results of a series of experiments to obtain laser action from an optically pumped bulk solution of high-gain laser dye (Rhodamine) solutions in strongly scattering media consisting of $\mathrm{TiO}_{2}$ nanoparticles initiated by Lawandy's group [16]-[20], independent of the studies of the above microspherical cavities. It is pointed out that multiple light scattering in a highly scattering and diffusive medium can trap excited photons as well as emitted photons in a small spatial region so that the gain exceeds the loss [16], [17]. Moreover, they mentioned that the high-gain laser dye scattering system produces narrowlinewidth emission without any additional reflections [21].

For the case of a micrometer-sized particle medium, it could be expected that discrete stationary modes owing to its distributed feedback-like configuration will improve such a high-gain dye laser performance. About a decade ago, Chang et al. demonstrated experimentally for the first time, to our knowledge, this fact by employing Rhodamine $6 \mathrm{G}$ (RH6G)-methanol solution containing spread layers of $\mathrm{OH}$-gel particles of about $5 \mu \mathrm{m}$ in diameter [22]. Appreciably narrowed linewidth $(\sim 0.8 \mathrm{~nm})$ together with sharp output-beam pattern, compared with that $(\sim 10 \mathrm{~nm})$ in amplified spontaneous emission from the dye in neat methanol, were confirmed employing a frequency-doubled, $Q$-switched Nd:YAG laser for the pumping.

Most recently, our first report on the results of laser action of the RH6G dye microdroplet (not from bulk solution) mixed with highly scattering particulates was presented [23], accompanying well-defined thresholds along with significantly increased emission intensities (not amplified spontaneous emission). In this experiment, we used the poly(methyl methacrylate) (PMMA) solid nanoparticles. 
In view of highly scattering and diffusive media producing multiple light scattering, a fat emulsion Intralipid is very interesting and useful. Intralipid is known to be used clinically as an intravenously administered nutrient, but sometimes it is employed for providing the scattering medium in a tissue phantom to investigate propagation of light in biological tissues [24]-[26] and optical imaging experiments [27], [28]. Though only one measurement of Raman spectroscopy was performed to determine diamond location in this medium [29], no lasing or SRS studies of immersed or mixed materials in this useful medium have been reported yet, as far as we are aware, not only in microspheres but also in bulk solutions.

In the present paper, we report experimental studies on lasing behaviors from dye-doped microdroplets containing highly scattering Intralipid by changing both the concentrations of RH6G dye and Intralipid. Almost-no-lasing neat-dye droplets which have much higher or much lower dye-concentration are examined by adding suitably fat emulsion Intralipid solution. Thus, well-defined lasing thresholds are observed when certain amounts of Intralipid solution are mixed to the neat dyedoped microdroplets. It is found evidently that Intralipid turbid solution mixed to microdroplets in neat-dye lasing at nearly optimum dye concentration is also remarkably effective for increment of peak intensity for laser emission. Hence, the effectiveness of Intralipid turbid medium is demonstrated to improve or to enforce the conventional (neat) dye laser characteristics just like the solid nanoparticles such as $\mathrm{TiO}_{2}$ and PMMA reported up to the present [16], [21], [23].

Moreover, the measurements of spatial distribution of emitted light and mode structures of lasing spectra from the dye microdroplets containing Intralipid scattering media are performed. These results are indicative of the disappearance of the well-known mode structures due to MDR's of spherical cavities depending on the substituted contents of Intralipid. Here, we should also point out that such dyeIntralipid mixtures, with soft scatterers in contrast to those previous solid nanoparticles, will be very interesting in relation with biocolloids in biological tissues and cellular structures.

\section{EXPERIMENTAL}

The experimental arrangement, as shown in Fig. 1, is similar to those described previously [7], [11], [12], [23]; it employs a frequency-doubled, $Q$-switched Nd:YAG laser [Nd:YAG second harmonic $(\mathrm{SH}), 532 \mathrm{~nm}$ ] with $\sim 10$-ns pulse duration, and then its output beam is focused onto dye-doped liquid microdroplets that scatter elastic $(532 \mathrm{~nm})$ and inelastic (redshifted) light into an optical multichannel analyzer. The weakly focused pump-beam size ( $\sim 450-\mu$ m diameter) was larger than the dye (RH6G)-doped microdroplet. Light emitted from the dye-doped microdroplet irradiated by the laser are collected at $90^{\circ}$ to the incident pump beam, and its spectrum is analyzed with an optical multichannel analyzer (OMA) mounted on a microscope. We formed proper-sized microdroplets around a fine horizontally strung tungsten fiber $(\sim 5-\mu \mathrm{m}$ diameter) by a simple method that inserts the fiber and then pulls back the fiber into and then from a water and glycerol solution containing RH6G dye. These RH6G-doped solutions are made
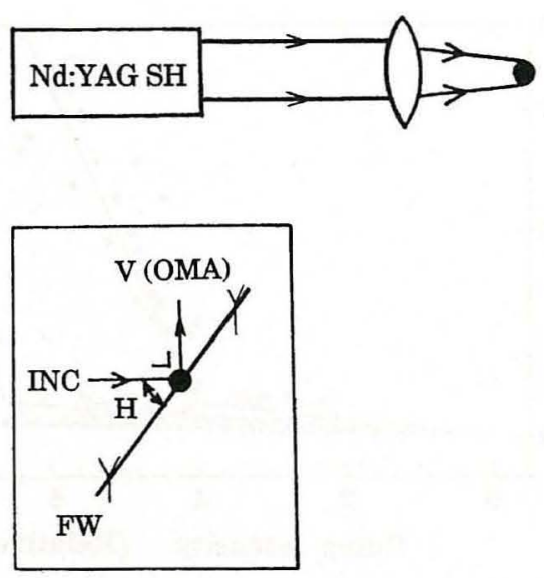

Fig. 1. Schematic diagram of the experimental arrangement. Nd:YAG SH: second harmonic $(532 \mathrm{~nm})$ of Nd:YAG laser for dye-doped microdroplet pumping; FW: fine tungsten wire strung in the horizontal plane $(\mathrm{H})$; OMA: optical multichannel analyzer viewing from the vertical plane (V), normal to the incident pump beam (INC).

from a water and glycerol (WG) mixture with a volume ratio of $\sim 10: 1$ for adequate viscosity.

We studied experimentally the optical properties with a variety of concentrations of the RH6G-doped spherical microdroplets (with diameter $D \simeq 100 \pm 5 \mu \mathrm{m}$ ) containing various amounts of Intralipid solution (Intralipid-10\%, manufactured by Kabi Pharmacia AB, Uppsala, Sweden). Various contents of the Intralipid-10\% in the RH6G-doped WG-solution were prepared by changing volume ratios of the WG and the Intralipid-10\% solutions.

\section{RESULTS AND Discussion}

\section{A. Pump-intensity Dye Microdroplet-Laser \\ Emission-Intensity Characteristics}

1) Dye Concentration Dependence Fig. 2 shows the emission intensity from the RH6G-doped single droplet at the peak wavelength, as a function of the relative pump-beam intensity $E_{p}$, as a parameter of RH6G-dye concentrations, without containing the scattering Intralipid-10\% (i.e., from the neat RH6G droplets): $1 \times 10^{-5} \mathrm{M}$ (plot A), $1 \times 10^{-4} \mathrm{M}$ (B), and $1 \times 10^{-3} \mathrm{M}(\mathrm{C})$ in Fig. 2(a), and $1 \times 10^{-3} \mathrm{M}$ (plot C), $2.5 \times 10^{-3} \mathrm{M}$ (D), and $5 \times 10^{-3} \mathrm{M}$ (E) in Fig. 2(b). In the neat RH6G microdroplets without the Intralipid, the cases of our lowest (plot $\mathrm{A}$ ) or highest (plot E) dye concentration exhibit a nearly linear slope in about $E_{p}<7$, or $E_{p}<8$ in our nearly maximum pump intensities, respectively, whereas the other microdroplets with the medium dye concentrations (plots B-D) reveal the drastic slope change, demonstrating the lasing thresholds at the bending points $\left[E_{p o} \simeq 6.7\right.$ (B), 4.0 (C), and 6.3 (D)]. We remark that a symptom of lasing threshold was observable at the much higher pump intensity $\left(E_{p}>7\right)$ in the much lower neat dye concentration (plot A), and no laser action could be obtained in the much higher neat dye concentration (plot E) to resemble our previous results using the PMMA solid-nanoparticles in the RH6G-doped single microdroplet [23]. 


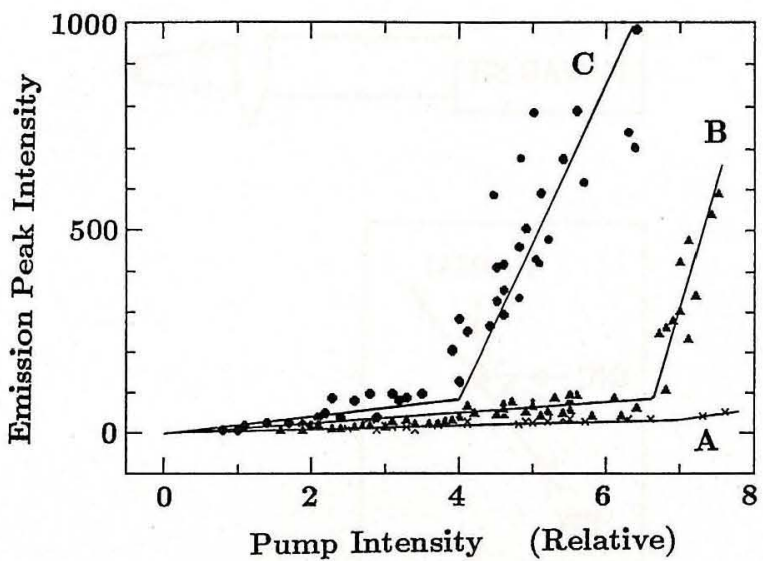

(a)

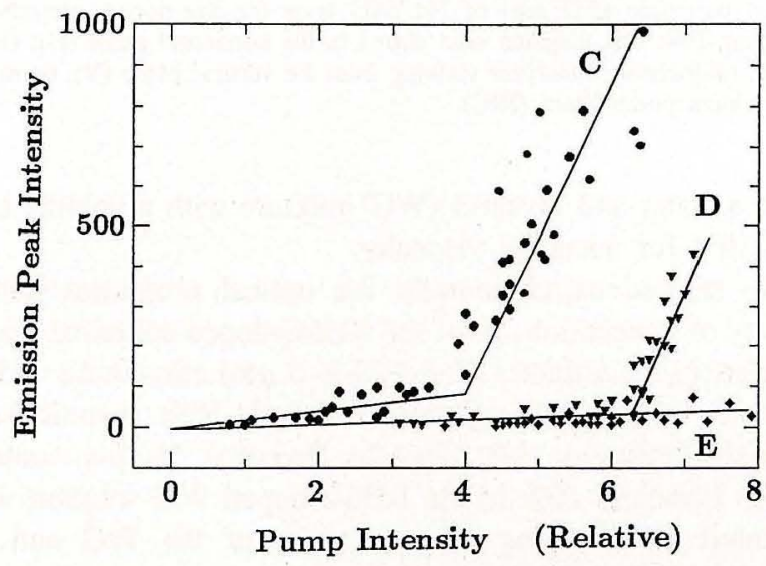

(b)

Fig. 2. Dye concentration dependence of the emission intensity from RH6G microdroplets, as a function of the relative pump-beam intensity $E_{p}$, without containing the scattering Intralipid-10\% (i.e., from the neat RH6G droplets). A: $1 \times 10^{-5}$ M, B: $1 \times 10^{-4} \mathrm{M}$, and C: $1 \times 10^{-3} \mathrm{M}$, in Fig. 2(a), and C: 1 $\times 10^{-3} \mathrm{M}, \mathrm{D}: 2.5 \times 10^{-3} \mathrm{M}$, and E: $5 \times 10^{-3} \mathrm{M}$, in Fig. 2(b), respectively.

\section{2) Effects of Intralipid-10\% Content Under Fixed Dye Con-} centrations Fig. 3 illustrates the emission intensity from the RH6G-doped single microdroplet at the peak wavelength as a function of the relative pump-beam intensity $E_{p}$, as a parameter of volume contents $\left(N_{c} \%\right)$ of the scattering Intralipid-10\%: $N_{c}=0 \%$ (plot A), $\sim 2 \%$ (B), 18\% (C), and $50 \%$ (D), under a fixed much higher dye concentration $(5 \times$ $10^{-3} \mathrm{M} \mathrm{RH6G)}$. The neat droplet without the Intralipid (plot A) shows a tendency for an approximately linear dependence of input-output intensities as indicated by $\mathrm{E}$ in Fig. 2(b), while the microdroplets containing the certain amounts of the Intralipid (plots B-D) provide two distinct regions with bending points corresponding to the lasing thresholds $\left[E_{p o} \simeq\right.$ $5.2(\mathrm{~B}), 4.4(\mathrm{C})$, and $5.6(\mathrm{D})]$.

On the other hand, for a much lower RH6G-dye concentration $\left(1 \times 10^{-5} \mathrm{M}\right)$, the analogous data to Fig. 3 are plotted in Fig. 4, compared with the neat dye $\left(N_{c}=0 \%\right.$ Intralipid$10 \%$ ) WG solution (plot A), $0.02 \%$ (B), $0.2 \%$ (C), $2 \%$ (D), and $4 \%(\mathrm{E})$. The RH6G neat droplet without the Intralipid (plot A) exhibits a nearly linear slope in about $E_{p}<7$ of input-output intensities as depicted by $\mathrm{A}$ in Fig. 2(a), whereas the microdroplets with Intralipid (plots B-E) reveal the lasing

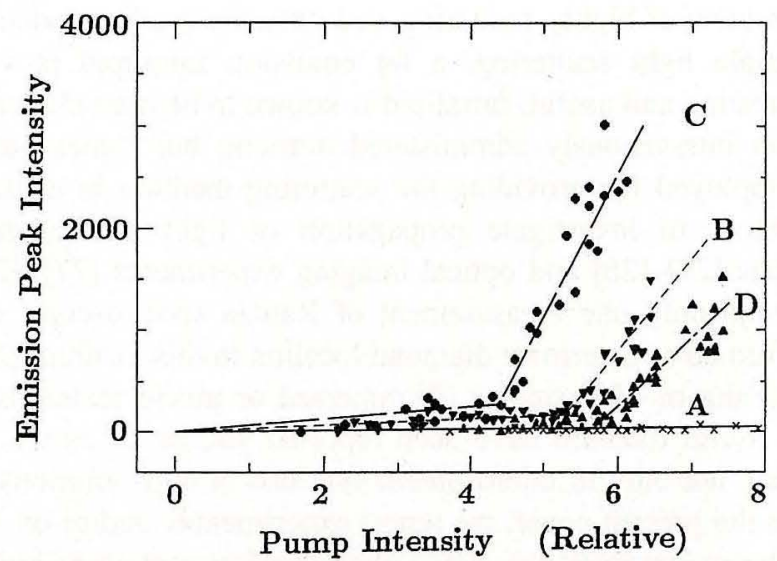

Fig. 3. Emission intensity from RH6G microdroplets $\left(5 \times 10^{-3} \mathrm{M}\right)$ containing Intralipid as a function of the relative pump-beam intensity $E_{p}$, as a parameter of volume contents $\left(N_{c} \%\right)$ of the scattering Intralipid-10\%. A: $N_{c}=0 \%$ (neat dye), B: $\sim 2 \%, \mathrm{C}: 18 \%$, and D: $50 \%$ under a fixed much higher dye concentration $5 \times 10^{-3} \mathrm{M}$ RH6G.

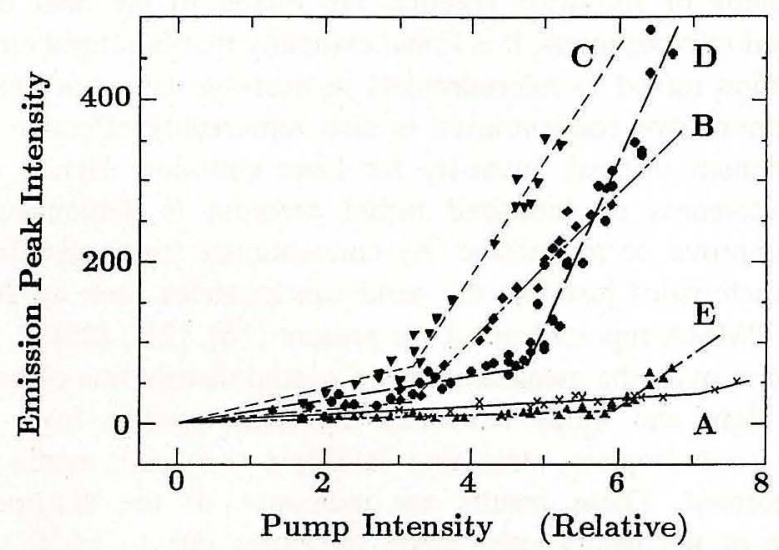

Fig. 4. Emission intensity from RH6G microdroplets $\left(1 \times 10^{-5} \mathrm{M}\right)$ containing Intralipid as a function of the relative pump-beam intensity $E_{p}$, as a parameter of volume contents $\left(N_{c} \%\right)$ of the scattering Intralipid-10\%. A: $N_{c}=0 \%$ (neat dye), B: $\sim 0.02 \%$, C: $0.2 \%, \mathrm{D}: 2 \%$, and E: $4 \%$, under a fixed much lower dye concentration of $1 \times 10^{-5} \mathrm{M}$ RH6G.

thresholds at the bending points $\left[E_{\text {po }} \simeq 3.2(\mathrm{~B}), 3.2(\mathrm{C}), 4.7\right.$ (D), and $6.0(\mathrm{E})]$. No effects of highly scattering nanoparticles on these much lower dye concentration solutions (even for bulk as well as for microdroplets) have been described, to the best of our knowledge.

Furthermore, with similar measurements to Figs. 3 and 4, Fig. 5 shows the case of the nearly optimum RH6Gdye concentration $\left(1 \times 10^{-3} \mathrm{M}\right)$ as illustrated in Fig. 2, in which the RH6G neat droplet without the Intralipid (plot A; $N_{c}=0 \%$ Intralipid-10\%) exhibits also the lasing threshold at the bending point $\left(E_{p o} \simeq 4.0\right)$, as well as the cases with the Intralipid: $N_{c} \simeq 2 \%$ (B), $18 \%$ (C), and $33 \%$ (D). Although no distinctive differences are observed with respect to the lasing thresholds in this microdroplet in such a nearly optimum dye concentration, the mixing of the Intralipid scatterers contributes remarkably to the enhancement of the microdroplet laser emission (in Fig. 5, emission intensity in plot $\mathrm{C}$ is approximately six times stronger than that in plot $\mathrm{A}$ at $E_{p} \simeq 6$ ). 


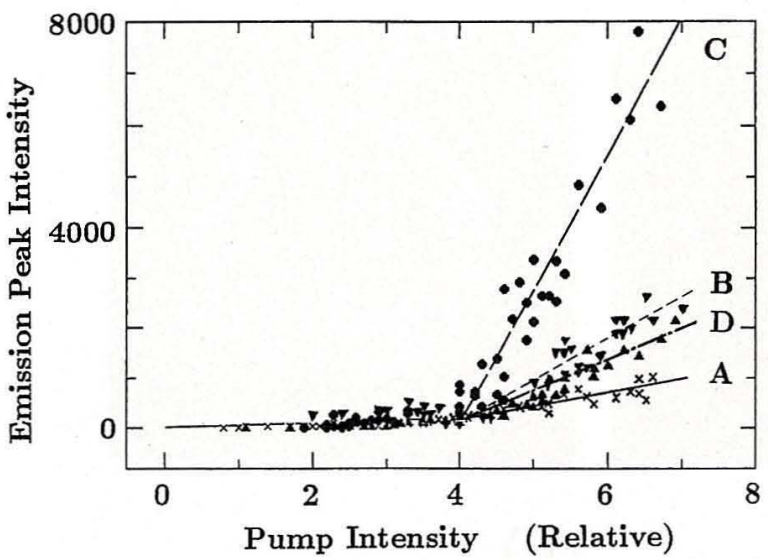

Fig. 5. Emission intensity from RH6G microdroplets $\left(1 \times 10^{-3} \mathrm{M}\right)$ containing Intralipid as a function of the relative pump-beam intensity $E_{p}$, as a parameter of volume contents $\left(N_{c} \%\right)$ of the scattering Intralipid-10\%. A: $N_{c}=0 \%$ (neat dye), B: $\sim 2 \%$, C: $18 \%$, and D: $33 \%$, under a fixed nearly optimum dye concentration of $1 \times 10^{-3}$ M RH6G.

It is noteworthy from the above results that optimum mixing ratios of the Intralipid-10\% to active dye solutions should exist for increasing the microdroplet-dye lasing intensity. For easy comparison of the main characteristics, Table I summarizes some typical results of measured lasing thresholds $\left(E_{p o}\right)$ and maximum emission intensity obtained from the microdroplets at $E_{p}=6$ with three RH-6G dye concentrations $5 \times 10^{-3}, 1$ $\times 10^{-3}$, and $1 \times 10^{-5} \mathrm{M}$, mixed with different contents of Intralipid-10\% solutions.

In general, it is understood that the maximum intensity of the emission peak and the lasing threshold with the dye and Intralipid mixture yield larger and lower values, respectively, than those in the respective neat dye, depending upon the dye concentration and the Intralipid-10\% content; this feature indicates the existence of the optimum content of Intralipid$10 \%$ solution for respective neat dye concentration.

\section{B. Spatial Distribution of Light Emitted from Dye Microdroplets}

We measured microscope images of spatial distribution of emission from the suspended single RH6G microdroplets with different amounts of Intralipid-10\% scatterers. Fig. 6 shows a comparison of observed image examples for the case of RH6G neat-dye concentration of $1 \times 10^{-3} \mathrm{M}$. This concentration corresponds to nearly optimum so that it exhibits the lasing threshold even without Intralipid scatterers (see Fig. 5). In these microscope photographs, the lower and upper ones correspond to the cases of the large amounts of the Intralipid$10 \%$ (volume content $\left.N_{c} \simeq 18 \%\right)$ and the neat dye $\left(N_{c}=\right.$ $0 \%$ ); Fig. 6(a) is the microdroplet image taken by whitelight illumination with the microscope, and Fig. 6(b) and (c) are, respectively, the images in fluorescence (below threshold, $E_{p} \simeq 1.0-3.0$ in Fig. 5) and in lasing (above threshold, $E_{p} \simeq$ 6.0-7.0 in Fig. 5), by Nd:YAG SH $(532 \mathrm{~nm})$ pumping. The exposure in Fig. 6(c) is reduced to about 1/20 times that in (b). Above the lasing threshold (c), the image of the emission pattern with the dye-Intralipid mixture [the lower figure of (c)] reveals rather extended, uniform character accompanied
TABLE I

Lasing Characteristics From Dye-Doped Microdroplets WITH/WITHOUT INTRALIPID-10\% SOLUTION MIXTURE

\begin{tabular}{cccc}
\hline $\begin{array}{c}\text { RH-6G Dye } \\
\text { Conc. (M) }\end{array}$ & $\begin{array}{c}\text { Intralipid-10\% } \\
\text { Contents (\%) }\end{array}$ & $\begin{array}{c}\text { Lasing Threshold } \\
\text { Epo (Relative) }\end{array}$ & $\begin{array}{c}\text { Max. Emission Peak } \\
\text { Ep=6 (Relative) }\end{array}$ \\
\hline $5 \times 10^{-3}$ & 0 & - & $(40)$ \\
& 2 & 5.2 & 850 \\
& 18 & 4.4 & 2600 \\
& 50 & 5.6 & 260 \\
$1 \times 10^{-3}$ & 0 & 4.0 & \\
& 2 & 4.0 & 880 \\
& 18 & 4.0 & 1800 \\
& 33 & 4.0 & 5500 \\
& & & 1400 \\
$1 \times 10^{-5}$ & 0 & 7.0 & $(30)$ \\
& 0.02 & 3.2 & 290 \\
& 0.2 & 3.2 & 470 \\
& 2 & 4.7 & 330 \\
& 4 & 6.0 & 20 \\
\hline
\end{tabular}

with the rather brighter region around the center, in contrast to that in the neat-dye microdroplet, where the rim appears brighter than the center [the upper figure of Fig. 6(c)]. In such a highly scattering medium, the mode structures due to MDR's of spheres may disappear since the laser gain due to multiple light scattering inside the microsphere becomes dominant to exceed internal loss. Even in the cases of Fig. 6(a) and (b) in the lower pictures, one can observe the occurrence of the multiple light scattering event compared with the upper ones for the neat-dye sample.

In Fig. 7, the microscope pictures, analogous to Fig. 6, are compared for the case of $1 \times 10^{-5} \mathrm{M}$ RH6G single microdroplet. This dye concentration is much lower, as understood from Table I. which exhibits the relatively low emission intensity even with Intralipid-10\% scatterers (see Fig. 4). These microscope images of Fig. 7 show the various cases; the upper figures show (a) the neat $\left(N_{c}=0 \%\right)$ dye lasing [at $E_{p} \simeq 7.5$ in Fig. 4 (A)], (b) the no-lasing with $N_{c}=$ $4 \%$ [at $E_{p} \simeq 5.0$ in Fig. 4 (E)], and (c) the lasing with $N_{c}=$ $0.02 \%$ [at $E_{p} \simeq 6.0$ in Fig. 4 (B)]. In the lower figures for the case of $N_{c} \simeq 0.2 \%$ [in Fig. 4 (C)], Fig. 7(a) corresponds to no-lasing and (b) and (c) to lasing, respectively [(a) at $E_{p} \simeq$ 2.0, (b) at $E_{p} \simeq 3.5$, and (c) at $E_{p} \simeq 4.5$ in Fig. 4 (C)]. The exposures in the upper figure of Fig. 7(a) and (c) are reduced to about 1/150 times that in the upper figure of Fig. 7(b), and those in the lower (b) and (c) are about 1/200 times that in the lower (a). Above the lasing threshold, the images of the emission pattern with the dye-Intralipid mixture [the upper (c) and the lower (b) and (c)] appear to be rather close to that in the neat droplet [the upper (a)]; that is somewhat different from the patterns as indicated in Fig. 6, in which the larger amounts of Intralipid are contained in the microdroplets.

\section{Mode Structures of Lasing Spectra from Dye Microdroplets}

From the above results showing microscope images of spatial distribution of emission from the suspended single RH6Gmicrodroplets containing different amounts of Intralipid-10\% 


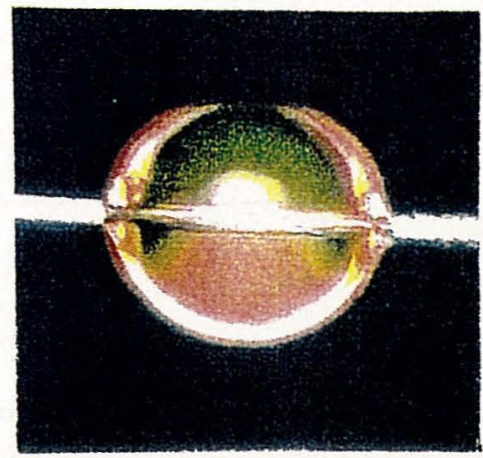

(a)

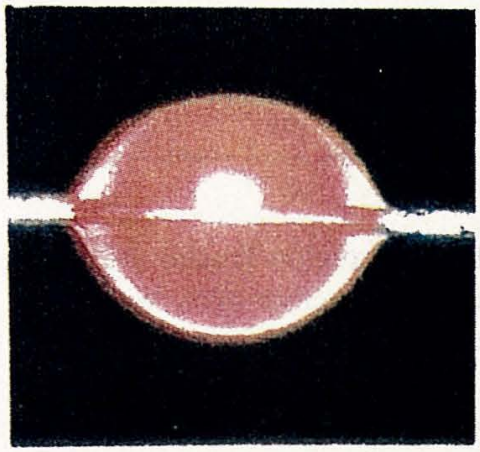

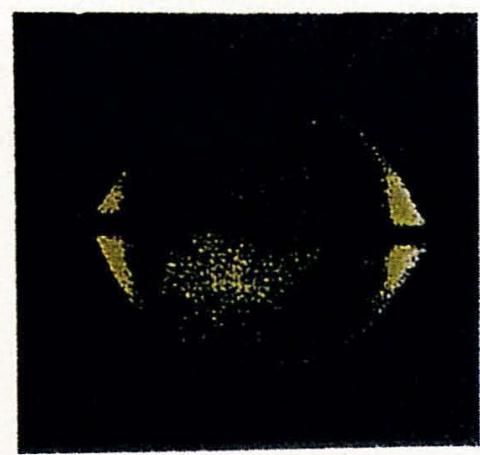

(b)

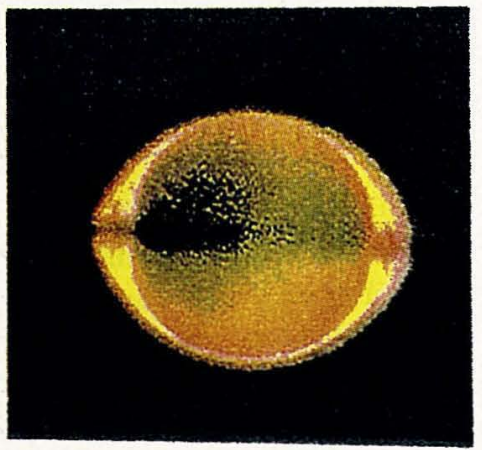

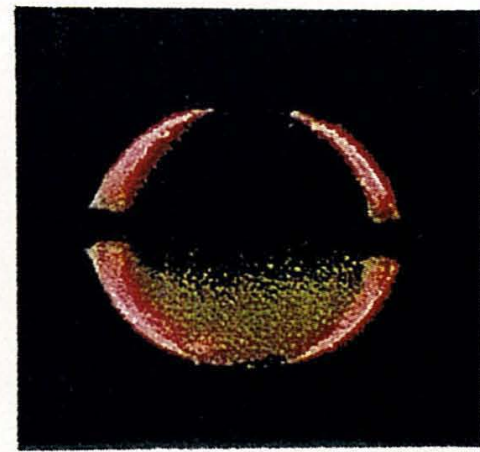

(c)

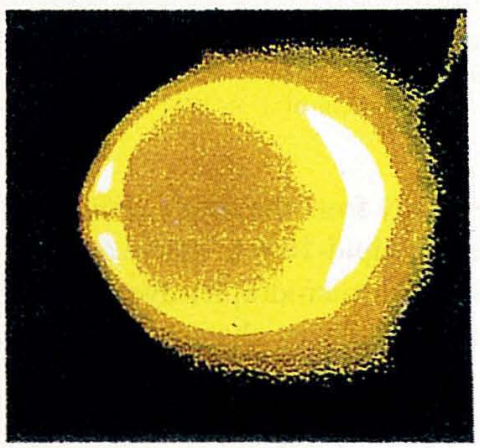

Fig. 6. Comparison of the microscope images of spatial distribution of light emission from the suspended single $\mathrm{RH} 6 \mathrm{G}-\mathrm{microdroplets}\left(1 \times 10^{-3} \mathrm{M}^{\mathrm{RH}} 6 \mathrm{G}\right)$ with different amounts of Intralipid-10\% scatterers. The lower and upper figures correspond to the cases in the large amounts of the Intralipid-10\% (volume content $N_{c} \simeq 18 \%$ ) and in the neat dye $\left(N_{c}=0 \%\right.$ ), respectively: (a) microdroplet image taken by white-light illumination with the microscope, and (b) and (c) are, respectively, the images in fluorescence (below threshold, $E_{p} \simeq 1.0-3.0$ in Fig. 5), and in lasing (above threshold, $E_{p} \simeq 6.0-7.0$ in Fig. 5), by Nd:YAG SH $(532 \mathrm{~nm})$ pumping. The exposure of (c) is about $1 / 20$ of (b).

scatterers, it is considered that the variations of mode structures may occur in these microdroplets. To the best of our knowledge, no studies on the changes of mode structures in microdroplet dye lasing containing scattering nanoparticles have been presented. The conjecture that mode structures should vary (from normal MDR mode structures) depending on the amounts of scattering nanoparticles motivated the following studies. The microdroplet diameter used in the above experimental measurements was relatively larger, which was that of near resolution limit in our spectral measurements $(\sim 1$ $\mathrm{nm}$, which corresponds to $\sim 100-\mu$ m diameter in the RH6G dye-lasing region). Hence, we examined mode structures of spectra emitted from RH6G-doped microdroplets with smaller diameters $D \leq 60 \mu \mathrm{m}$, to be within the restriction of our OMA resolution.

All of the figures below show the emission spectra of lasing (from the RH6G-doped single microdroplets) as a parameter of the relative pump intensity $E_{p}$ with the comparison for two different microdroplet diameters $D$; (a) $\sim 60 \mu \mathrm{m}$ (the left), and (b) $\sim 30 \mu \mathrm{m}$ (the right).

Fig. 8 illustrates the measured spectra for neat droplet $\left(10^{-3}\right.$ M RH6G) without the Intralipid-10\% that exhibits almost an appearance of the well-known periodic mode structures [3], [4], owing to MDR's of spherical cavity [mode spacing $\Delta \lambda \simeq 1.5$ and $3 \mathrm{~nm}$ in (a) and (b), for $D \simeq 60$ and $30 \mu \mathrm{m}$, respectively, where the refractive index of the sphere $m \simeq$
1.33 for water is assumed]. The center wavelength of lasing is situated at $\lambda_{c} \simeq 610-615 \mathrm{~nm}$. The MDR mode structures in the lower $E_{p}$ (the lower spectra) seem to be rather intermittent, compared with those in the higher $E_{p}$ (the upper spectra). Substructures of modes are also seen.

The case of $N_{c}=18 \%$ with the larger contents of the Intralipid-10\% at the same dye concentration as Fig. $8\left(10^{-3}\right.$ M RH6G) is shown in Fig. $9\left(\lambda_{c} \simeq 585-590 \mathrm{~nm}\right)$, in which the MDR mode structures disappear; the spiky structures appearing in Fig. 9(b) are clearly distinguishable from those owing to MDR's in Fig. 8.

Moreover, Fig. $10\left(\lambda_{c} \simeq 610-615 \mathrm{~nm}\right)$, similar to Figs. 8 and 9 , comes from the case of $N_{c} \simeq 2 \%$ with the smaller contents of the Intralipid-10\% at the same dye concentration as Figs. 8 and $9\left(10^{-3} \mathrm{M}\right.$ RH6G). There exists a tendency of mode structures owing to MDR's in Fig. 10(b) at lower pump intensities, while there are no mode structures, as yet, in Fig. 10(a) at higher pump intensities.

Different from the dye concentration in Figs. 8-10, Fig. 11 ( $\lambda_{c} \simeq 585-590 \mathrm{~nm}$ ) is the other lasing spectra in the case of $N_{c} \simeq 0.2 \%$ with the lower RH6G concentration $\left(10^{-5} \mathrm{M}\right.$ RH6G). The mode structures in Fig. 11 considerably resemble those owing to MDR's in the neat dye lasing (Fig. 8), although the mode structures in Fig. 11(a) seem to be somewhat intermittent compared with those in Fig. 8(a). In Fig. 11(a), it is seen that the other lasing spectra (although almost no 


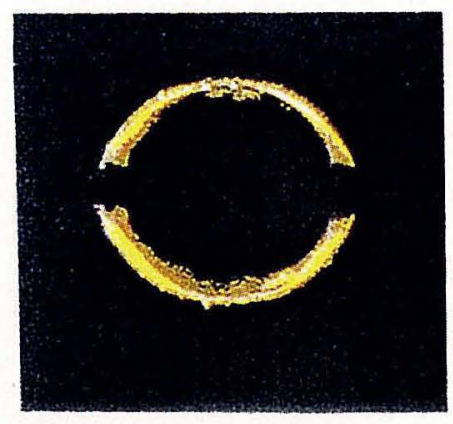

(a)

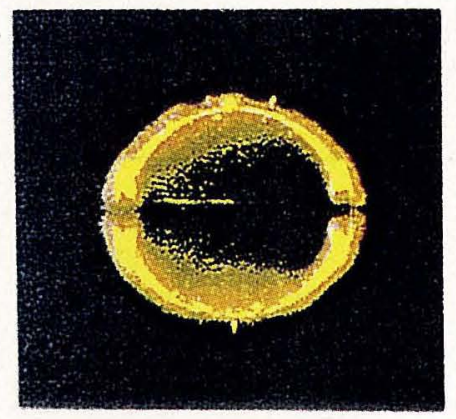

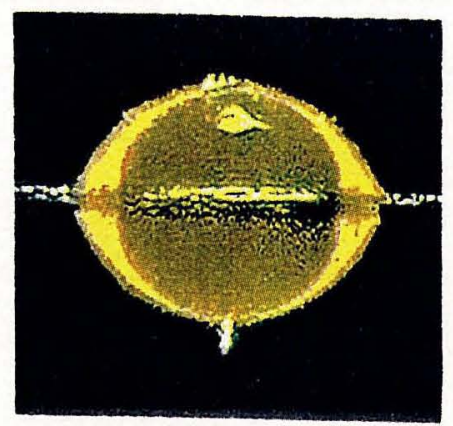

(b)

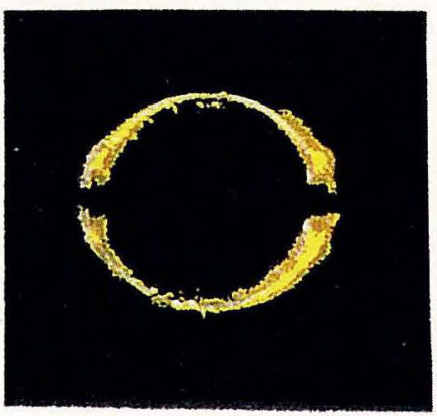

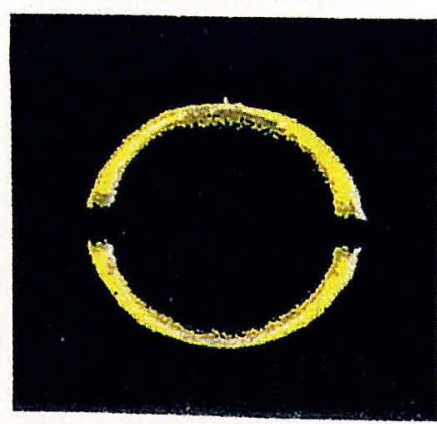

(c)

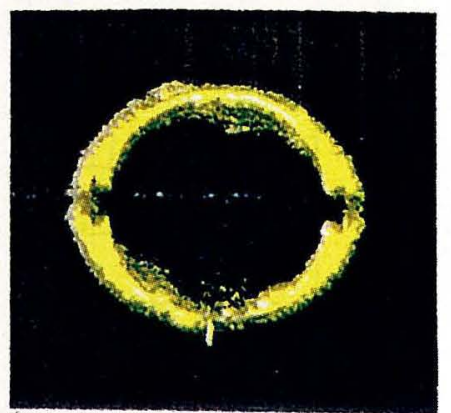

Fig. 7. Microscope images analogous to Fig. 6 , for $1 \times 10^{-5}$ M RH6G. In the upper figures, (a) the neat $\left(N_{c}=0 \%\right)$ dye lasing [at $E_{p} \simeq 7.5$ in Fig. 4 (A)], (b) the no-lasing with $N_{c}=4 \%$ [at $E_{p} \simeq 5.0$ in Fig. 4 (E)], and (c) the lasing with $N_{c}=0.02 \%$ [at $E_{p} \simeq 6.0$ in Fig. 4 (B)]. In the lower figures, all containing Intralipid $N_{c}=0.2 \%$ [in Fig. 4 (C)], (a) corresponds to the no-lasing [at $E_{p} \simeq 2.0$ ], (b) lasing [at $E_{p} \simeq 3.5$ ], and (c) lasing [at $E_{p} \simeq 4.5$ ]. The exposures of the upper (a) and (c) are about $1 / 150$ of the upper (b), and those of the lower (b) and (c) are about $1 / 200$ of the lower (a).

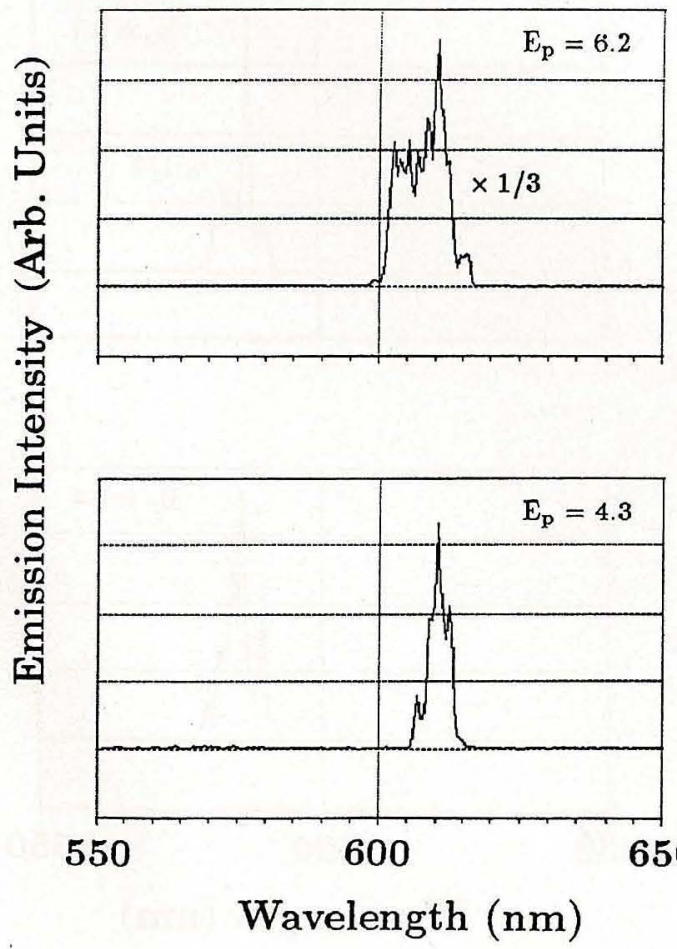

(a)
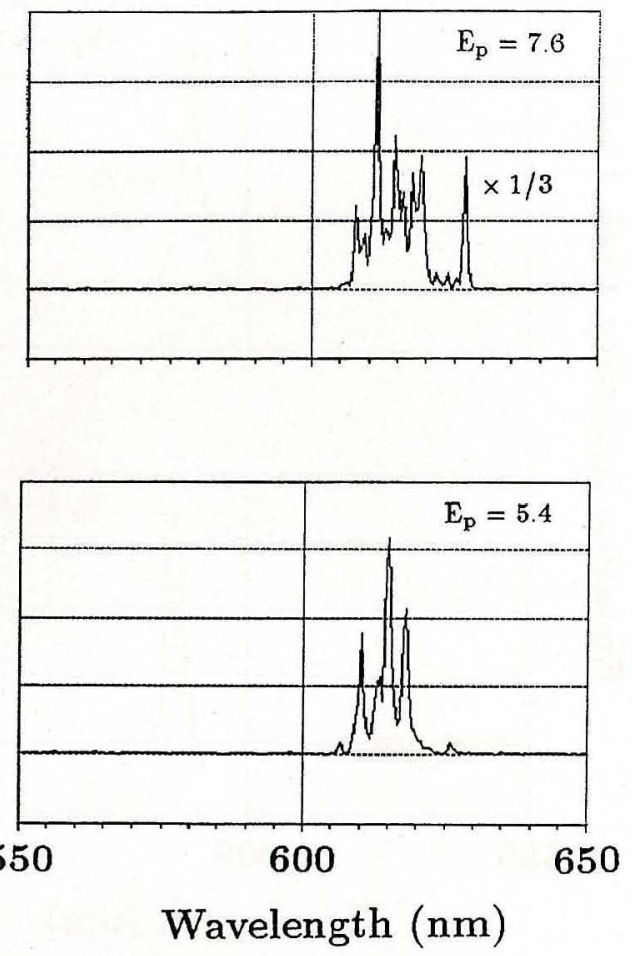

(b)

Fig. 8. Typical emission spectra from the RH6G neat microdroplet $\left(1 \times 10^{-3} \mathrm{M}\right.$ RH6G) without the Intralipid-10\%. (a) and (b) show the cases for $D \simeq 60$ and $30 \mu \mathrm{m}$, respectively.

mode structures appear) build up in the shorter wavelengths $\left(\lambda_{c} \simeq 565 \mathrm{~nm}\right)$, in which the laser gain of the shorter $\left(\lambda_{c} \simeq 565 \mathrm{~nm}\right)$ and longer wavelengths $\left(\lambda_{c} \simeq 585 \mathrm{~nm}\right)$ is inverted, depending on the pumping intensity $E_{p}$. Such a gain inversion may be possible in the microdroplet dye lasing accompanying with blue-shifts according to the experiment 


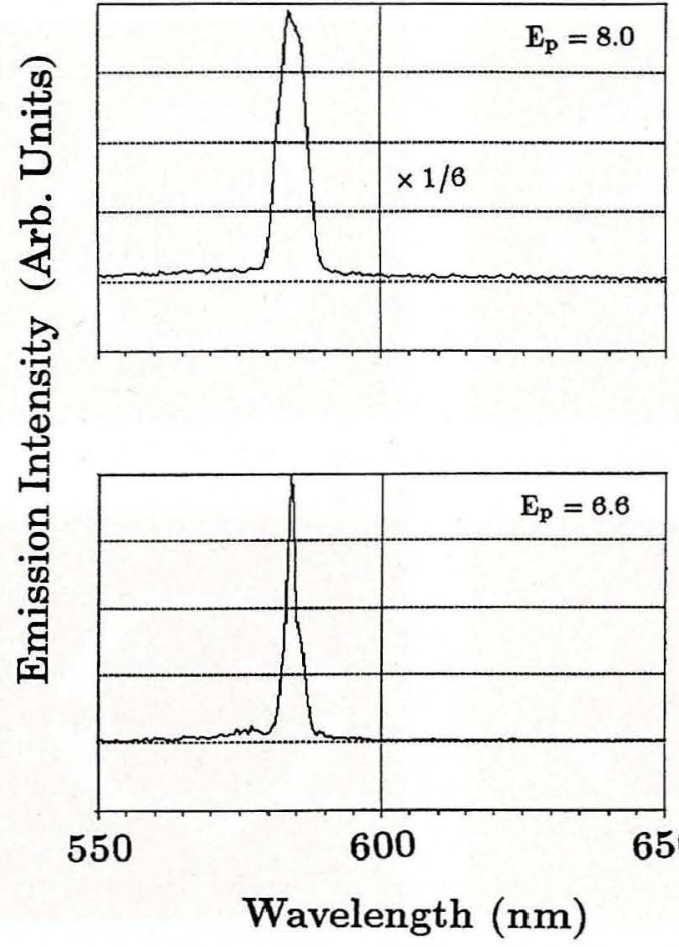

(a)
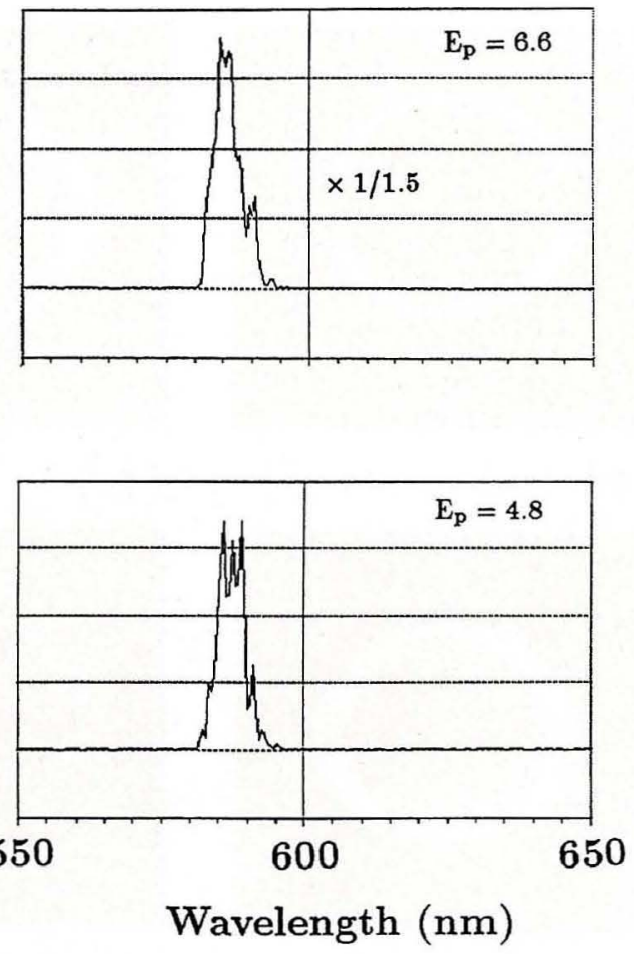

(b)

Fig. 9. Measured emission spectra from RH6G microdroplets with Intralipid-10\% contents of $N_{c}=18 \%$ at the same dye concentration as Fig. 8 ( $1 \times$ $10^{-3} \mathrm{M} \mathrm{RH6G).} \mathrm{(a)} D \simeq 60$ and (b) $30 \mu \mathrm{m}$, respectively.

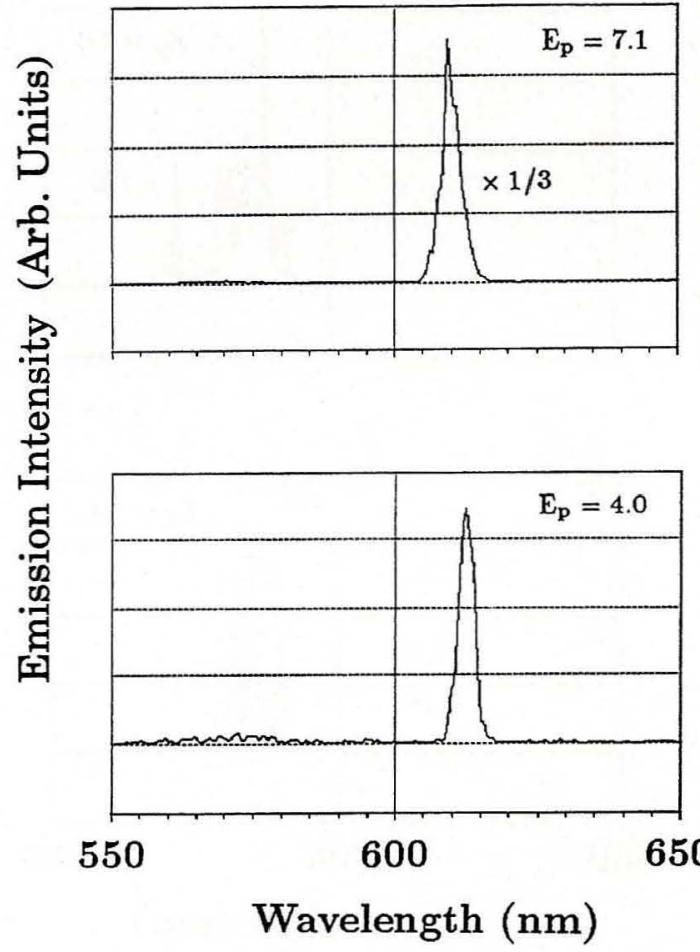

(a)
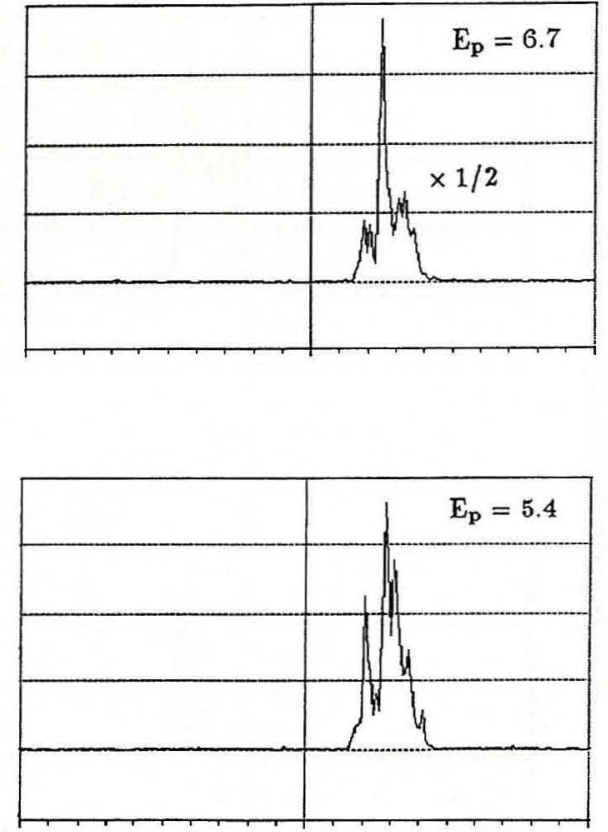

600 650

(b)

Fig. 10. Measured emission spectra from RH6G microdroplets with Intralipid-10\% contents of $N_{c} \simeq 2 \%$ at the same dye concentration as Figs. 8 and $9\left(1 \times 10^{-3} \mathrm{M}\right.$ RH6G). (a) $D \simeq 60$ and (b) $30 \mu \mathrm{m}$, respectively.

employing microdroplet dye-containing nanoscatterers [23], like that in bulk [18], in which the wavelength dependence of gain inversion is inverse to the present one.
In general, the spectral width of lasing broaden as the pumping intensity increases [exceptionally, Fig. 10(b) shows the inverse variation of the spectral width accompanied with 


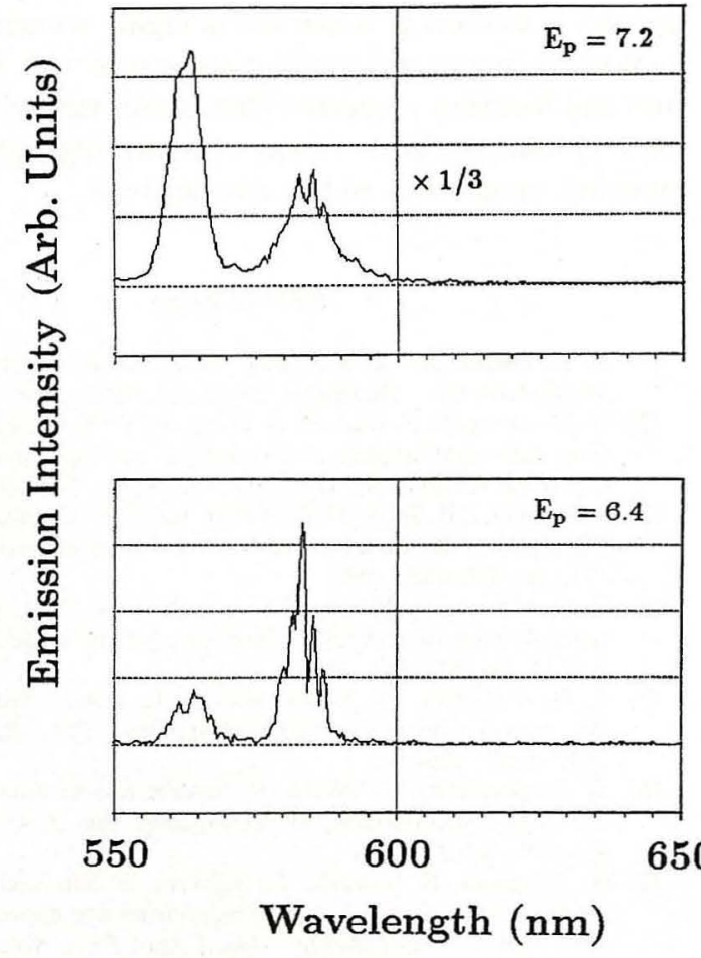

(a)
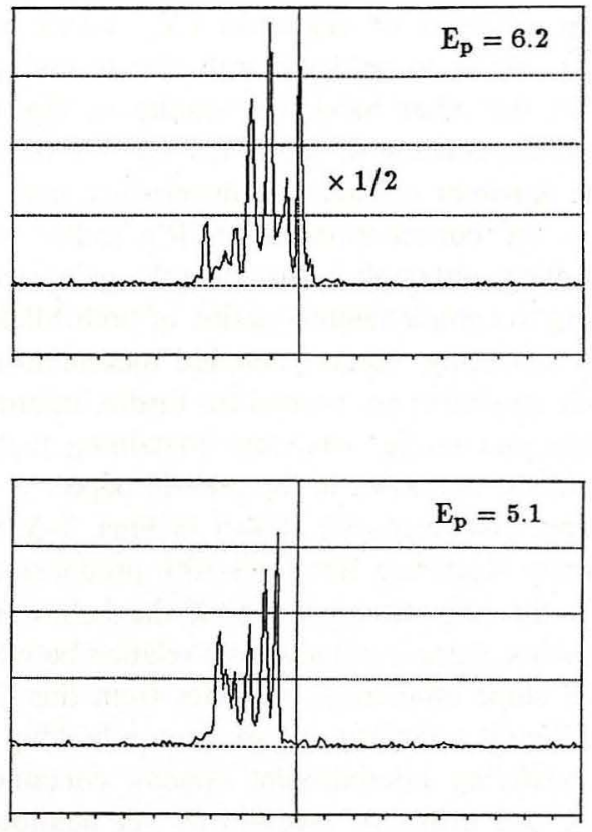

600

650

(b)

Fig. 11. Measured emission spectra from RH6G microdroplets with Intralipid-10\% contents of $N_{c} \simeq 0.2 \%$ at the lower RH6G concentration $\left(1 \times 10^{-5}\right.$ M RH6G), which is different from that in Figs. 8-10. (a) $D \simeq 60$ and (b) $30 \mu \mathrm{m}$, respectively.

the strong build-up of the specific mode]. This fact also suggests that only the spectral narrowing is not a full condition to confirm the laser action in high-gain dye-microdroplet scattering system [23].

Moreover, our measurements show the larger blue-shifts of spectra for the case of the Intralipid mixing in addition to the known blue-shifts due to lowering in usual dye concentration similar to the previous result [23]. Such blueshift phenomena must be related to effective spectral overlap between absorption and emission of dyes in this dye-scatterer microsystem also, just same as that in a dye-alone system [30], [31]. We should also mention that even with the smaller microdroplets (e.g., $D \leq 60 \mu \mathrm{m}$ ) within the limit of our OMA resolution, a tendency of disappearing of the wellknown periodic mode structures owing to MDR's of spherical cavities occurs when substituted Intralipid-10\% quantities are increased to the neat-dye microdroplets possessing normal MDR structures. This tendency seems to be also recognized by microscope observation of spatial distribution of microdroplet dye emission as presented in Figs. 6 and 7.

As mentioned before in Section I, there recently have been some studies on the effect of scatterers on lasing behaviors in liquid droplets that contain fluorescent dye solution of mixed fluorescent sol, polystyrene spheres, and undoped latexsol particles [13]-[15]. Nevertheless, they were all involved only in the MDR-dominated cases because of the originally occurred stimulated emission, in which it is not mentioned that the seeded nanoparticles work to enhance the stimulated emission, in contrast to no originally occurred lasing cases of the present study. It is also mentioned in [13] that the scatterers induce the output coupling enhancement as a result of cavity $Q$ degradation of the droplets. However, this consideration gives us no reasonable explanation for gain increment due to extended optical path caused by multiple scattering inside highly scattering dye-mixed microdroplet from the no-lasing state of neat droplets to the lasing state of droplets with Intralipid scatterers.

It is considered that in the cases of dye-Intralipid mixture in much higher (Fig. 3) or much lower (Fig. 4) RH6G dye concentrations, optical gain contributed by multiple light scattering is much larger than that due to MDR's [Fig. 2(a) and (b)]. However, in the case of optimum dye concentration (Fig. 5), the gain due to MDR's in the neat microdroplet makes it possible to achieve the lasing threshold, but that provided by multiple light scattering in the dye-Intralipid mixture readily exceeds the former. In general, in neat droplets, laser intensity builds up through optical feedback owing to MDR's at the sphere-surrounding medium interface, while adding a scattering medium in the droplets should make it difficult to form MDR's, because the laser intensity may become more or less dominant depending upon the mixed concentration of the medium through multiple light scattering in a small spatial region, just same as those in bulk cells [16], [17]. Based on this consideration, we conjecture that there exists a competitive process in starting laser oscillation between MDR's and multiple light scattering that effectively extends the optical path in the dye-Intralipid mixture for the cases of the seeded microdroplets; the results of Fig. 8 in the neat droplets is certainly contributed to by the MDR's, whereas the results shown in Figs. 9 and 10, in the cases 
of relatively larger contents of scatterers $\left(N_{c}=18 \%\right.$ and $\sim 2 \%$, respectively), appear to be dominantly due to multiple light scattering. On the other hand, the results of Fig. 11, in the case of smaller content of scatterers $\left(N_{c} \simeq 0.2 \%\right)$, which exhibits the apparent resonant characteristics, may be dominantly due to the contribution of MDR's rather than multiple light scattering, although in this state the gain seems to be built up owing to complementary action of both MDR's and multiple light scattering. Hence, detailed measurements on spectral or mode structures are needed for further information of dye-microdroplet lasing behaviors containing highly scattering nanoparticles, as shown in the present paper.

In the input-output characteristics shown in Figs. 3-5, the addition of the highly scattering Intralipid-10\% produces the different effects in the respective regions of the below and above lasing thresholds; there is not always a relation between laser threshold and slope efficiency. We infer from this fact that there exist different contributions to photon-buildup in a multiple light scattering microdroplet system, containing a highly scattering and diffusive medium as yet unknown, between incoherently spontaneous (below thresholds) and coherently stimulated processes (above thresholds). For example, the emission intensities in the plot $\mathrm{D}$ in Fig. 4 (with the higher $E_{p o} \simeq 4.7$, compared with the case in the plot $C$ with the lower $E_{p o} \simeq 3.2$, as listed in Table I) are much lower than those in the plot $C$ in all $E_{p}$ (up to $E_{p} \simeq 6$ ), but, the slope in the plot $\mathrm{D}$ above the lasing threshold $\left(E_{p o} \simeq 4.7\right)$ is rather steeper than that in plot $\mathrm{C}$.

The bending curves in Figs. 2-5 exhibiting the slope change in the input-output characteristics provide the clear evidence for laser action [2], [5], [16], not amplified spontaneous emission [32]. Temporal characteristics (pulse-duration variation) against pump intensities are needed also for further information of microdroplet-lasing behaviors containing highly scattering nanoparticles such as Intralipid.

\section{CONCLUSION}

We have described and discussed the experimental results of lasing characteristics of dye-doped spherical microdroplets containing highly scattering Intralipid-10\% turbid solution producing multiple light scattering. It has been demonstrated that a well-defined lasing threshold is observable at certain (optimum) Intralipid mixing ratios to microdroplets in neat dye as well as appreciable enhancement of the emission peak intensity. Another noteworthy finding is a possible tendency of disappearance of MDR's character inherent to normal neat-dye microdroplets, which were examined by microscope images of spatial distribution as well as by spectral measurements of their emission light. Thus, the present novel method using highly scattering Intralipid medium could be widely applied not only to a large number of dyes and similar materials for enhancing or enforcing their laser action, but also to Raman spectroscopic technique [33] based on conventional and stimulated Raman scattering. We hope also that this technique is useful for highly sensitive optical detection from small-quantity species with weak signal intensities such as biocolloids and biomolecules and for extending the Raman spectroscopic method to identify small objects and species embedded or immersed in highly scattering media like biological tissues and cellular structures with substitution of soft and harmless scatterers. Hence, our method is expected to be very attractive for a variety of future applications including practical diagnostics and in vivo analysis.

\section{REFERENCES}

[1] P. W. Barber, and R. K. Chang, Eds., Optical Effects Associated With Small Particles. Singapore: World Scientific, 1988.

[2] H.-M. Tzeng, K. F. Wall, M. B. Long, and R. K. Chang, "Laser emission from individual droplets at wavelengths corresponding to morphologydependent resonances," Opt. Lett., vol. 8, pp. 499-501, 1984.

[3] S.-X. Qian, J. B. Snow, H.-M. Tzeng, and R. K. Chang, "Lasing droplets: Highlighting the liquid-air interface by laser emission," Science, vol. 231, pp. 486-488, 1986.

[4] H.-B. Lin, A. L. Huston, B. J. Justus, and A. J. Campillo, "Some characteristics of a droplet whispering-gallery mode laser," Opt. Lett., vol. 11, pp. 614-616, 1986.

[5] C. G. B. Garret, W. Kaiser, and W. L. Bond, "Stimulated emission into optical whispering modes of spheres," Phys. Rev., vol. 124, pp. $1807-1809,1961$

[6] M.-K. Gonokami, K. Takeda, H. Yasuda, and K. Ema, "Laser emission from dye-doped polystyrene microsphere," Jpn. J. Appl. Phys., vol. 31, pp. L99- L101, 1992.

[7] H. Taniguchi, H. Yamada, T. Fujiwara, S. Tanosaki, H. Ito, H. Morozumi, and M. Baba, "Laser emission from dye-doped small spheres by ultraviolet $\mathrm{N}_{2}$ laser pumping," Jpn. J. Appl. Phys., vol. 32, pp. L58-L61, 1993.

[8] J. B. Snow, S.-X. Qian, and R. K. Chang, "Stimulated Raman scattering from individual water and ethanol droplets at morphology-dependent resonances, Opt. Lett., vol. 10, pp. 37-39, 1985.

[9] S.-X. Qian and R. K. Chang, "Multi-order Stokes emission from micrometer-sized droplets," Phys. Rev. Lett., vol. 56, pp. 926-929, 1986.

[10] R. G. Pinnick, A. Biswas, P. Chylek, R. L. Armstrong, H. Latifi, E. Creegan, V. Srivastava, M. Jarzembski, and G. Fernandez, "Stimulated Raman scattering in micrometer-sized droplets," Opt. Lett., vol. 13, pp. 494-496, 1988.

[11] H. Taniguchi and S. Tanosaki, "Three-color whispering-gallery-mode dye lasers using dye-doped liquid spheres," Jpn. J. Appl. Phys., vol. 32, pp. L1421-L1424, 1993.

[12] H. Taniguchi and H. Tomisawa, "Simple arrangement for liquid-droplet experiments due to morphology-dependent resonances," Rev. Sci. Instrum., vol. 64, pp. 3594-3597, 1993.

[13] R. L. Armstrong, J.-G. Xie, T. E. Ruekgauer, and R. G. Pinnick, "Energy-transfer-assisted lasing from microdroplets seeded with fluorescent sol," Opt. Lett., vol. 17, pp. 943-945, 1992.

[14] H.-B. Lin, A. L. Huston, J. D. Eversole, A. J. Campillo, and P. Chylek, "Internal scattering effects on microdroplet resonant emission structure," Opt. Lett., vol. 17, pp. 970-972, 1992.

[15] R. L. Armstrong, J.-G. Xie, T. E. Ruekgauer, J. Gu, and R. G. Pinnick, "Effects of submicrometer-sized particles on microdroplet lasing," Opt. Lett., vol. 18, pp. 119-121, 1993.

[16] N. M. Lawandy, R. M. Balachandran, A. S. L. Gomes, and E. Sauvain, "Laser action in strongly scattering media," Nature, vol. 368, pp. 436-438, 1994.

[17] A. Z. Genack and J. M. Drake, "Scattering for super-radiation," Nature, vol. 368, pp. 400-401, 1994.

[18] W. L. Sha, C.-H. Liu, and R. R. Alfano, "Spectral and temporal measurements of laser action of Rhodamine 640 dye in strongly scattering media," Opt. Lett., vol. 19, pp. 1922-1924, 1994.

[19] N. M. Lawandy and R. M. Balachandran, "Lawandy and Balachandran reply," Nature, vol. 373, p. 204, 1995.

[20] W. Zhang, N. Cue, and K. M. Yoo, "Emission linewidth of laser action in random gain media," Opt. Lett., vol. 20, pp. 961-963, 1995.

[21] R. M. Balachandran and N. M. Lawandy, "Interface reflection effects in photonic paint," Opt. Lett., vol. 20, pp. 1271-1273, 1995.

[22] N. Chang, T. Suzuki, and H. Inaba, "Distributed feedback dye laser using small gel particle layer as a periodic structure," Oyo-Buturi, vol. 54, pp. 841-846, 1985 (in Japanese).

[23] H. Taniguchi, M. Nishiya, S. Tanosaki, and H. Inaba, "Lasing behavior in a liquid spherical dye laser containing highly scattering nanoparticles," Opt. Lett., vol. 21, pp. 263-265, 1996.

[24] H. J. van Staveren, C. J. M. Moes, J. van Marle, S. A. Prahl, and M. J. C. van Gemert, "Light scattering in Intralipid-10\% in the wavelength range of 400-1100 nm," Appl. Opt., vol. 30, pp. 4507-4514, 1991. 
[25] G. Muller, B. Chance, R. R. Alfano, S. Arridge, J. Beuthan, E. Gratton, M. Karchke, B. Masters, S. Svanberg, and P. van der Zee, Eds., Medical Optical Tomography: Functional Imaging and Monitoring. Bellingham, WA: SPIE Opt. Eng., 1993.

[26] R. R. Alfano, Ed., OSA Proc. on Advances in Optical Imaging and Photon Migration. Washington, DC: Opt. Soc. Amer., 1994, vol. 21.

[27] K. P. Chan, M. Yamada, and H. Inaba, "Micrometer-resolution, optical imaging of objects through highly scattering media using a heterodyne detector array," Electron. Lett., vol. 30, pp. 1753-1754, 1994.

[28] K. P. Chan, M. Yamada B. Devaraj, and H. Inaba, "Optical imaging through highly scattering media by use of heterodyne detection in the 1.3- $\mu \mathrm{m}$ wavelength region," Opt. Lett., vol. 20, pp. 492-494, 1995

[29] C. A. Thompson, J. S. Reynolds, K. J. Webb, F. P. LaPlant, and D. Ben-Amotz, "Raman spectroscopic studies of diamond in Intralipid," Opt. Lett., vol. 20, pp. 1195-1197, 1995.

[30] H. Taniguchi, H. Tomisawa, and Sarjono, "Morphology-dependent dye lasing from a single microdroplet with double-layered dye doping," Opt. Lett., vol. 19, pp. 366-368, 1994.

[31] M. M. Mazumder, G. Chen, R. K. Chang, and J. B. Gillespie, "Wavelength shifts of dye lasing in microdroplets: Effect of absorption change," Opt. Lett., vol. 20, pp. 878-880, 1995.

[32] D. S. Wiersma, M. P. van Albada, and A. Lagendijk, "Random laser?," Nature vol. 373, pp. 203-204, 1995.

[33] H. Taniguchi, Y. Sato, S. Tanosaki, and H. Inaba, "Continuous-wave stimulated Raman scattering from micrometer-sized liquid droplets realized by mixing of highly scattering intralipid," to be published.
Hiroshi Taniguchi, photograph and biography not available at the time of publication.

Shinji Tanosaki, photograph and biography not available at the time of publication.

Kazuhiro Tsujita, photograph and biography not available at the time of publication.

Humio Inaba (SM'65-F' 85 ), photograph and biography not available at the time of publication. 